\title{
Estudios de materiales de cátodos híbridos y ánodos vítreos. Caracterización en celdas de ion litio.
}

\author{
A.K. CUENTAS-GAllegos, ${ }^{1}$ M.R. PAlaCín, ${ }^{1}$ M.T. COlOMER, ${ }^{2}$ J.R. JURADO, ${ }^{2}$ P. GÓMEZ-ROMERO ${ }^{1 *}$ \\ 1) Instituto de Ciencia de Materiales de Barcelona (ICMAB-CSIC). \\ 2) Instituto de Cerámica y Vidrio (ICV-CSIC)
}

\begin{abstract}
Como parte de nuestros estudios de nuevos materiales de electrodos para aplicación en celdas reversibles de litio, hemos abordado el estudio de materiales vítreos e híbridos [1] como posibles alternativas a los materiales activos cristalinos, que ven frecuentemente limitada su capacidad como resultado de transiciones de fase irreversibles. Dentro de este trabajo se presentan aquí los resultados recientes sobre cátodos híbridos de $\mathrm{PPi} / \mathrm{MnO}_{2}\left(\mathrm{PPi}=\right.$ polipirrol) y de $\mathrm{PAni} / \mathrm{V}_{2} \mathrm{O}_{5}(\mathrm{PAni}=$ polianilina), y de ánodos basados en vidrios en el sistema V-Ni-Te-O, así como de su combinación en celdas reversibles de ion litio. Hemos logrado obtener mediante reacción directa de pirrol con permanganato el híbrido $\mathrm{PPi} / \mathrm{MnO}_{2}$, y hemos observado que en la síntesis de $\mathrm{PAni} / \mathrm{V}_{2} \mathrm{O}_{5}$ existen factores que influyen positivamente en su comportamiento electroquímico.
\end{abstract}

Palabras clave: ánodos vítreos, cátodos híbridos, baterías de litio, polimeros conductores, óxidos electroactivos.

Study of Hybrid Cathode Materials and Vitreous Anodes. Characterization in Lithium Ion Cells.

This paper is based on new materials applied as electrodes in rechargeable lithium batteries. We have approached the study of glassy and hybrid materials as an alternative to crystalline active materials, which capacity is frequently limited by irreversible phase transitions. We present here our latest results on hybrid cathodes, $\mathrm{PPy} / \mathrm{MnO}_{2}(\mathrm{PPy}=\mathrm{PPi}=$ polypirrol) and $\mathrm{PAni} / \mathrm{V}_{2} \mathrm{O}_{5}$ (PAni= polyaniline), and anodes based on glasses of $\mathrm{V}-\mathrm{Ni}-\mathrm{Te}-\mathrm{O}$, and their combination in reversible lithium ion batteries. We have obtained the PPy/ $\mathrm{MnO}_{2}$ hybrid by direct one-pot reaction of pyrrole and permanganate, and for the preparation of PAni $/ \mathrm{V}_{2} \mathrm{O}_{5}$ we have determined the positive influence of some parameters on the electrochemical behaviour.

Keywords: Glass anodes, hybrid cathodes, lithium batteries, conducting polymers, electroactive oxides.

\section{INTRODUCCIÓN}

La evolución de aparatos electrónicos portátiles (móviles, ordenadores etc) apunta claramente hacia el desarrollo de dispositivos con mayores prestaciones y menor tamaño. Este desarrollo requiere de baterías del mínimo tamaño con mayor densidad de energía. Actualmente las baterías recargables basadas en litio presentan las mejores prestaciones en este sentido. Entre los materiales activos de cátodo se han propuesto y están en fase de estudio numerosos tipos de compuestos, generalmente óxidos cristalinos que siguen la estela del $\mathrm{LiCoO}_{2}$ empleado por Sony en sus baterías comerciales. Sin embargo, existen otras varias alternativas, entre las que destacan el uso de compuestos amorfos, en los que la falta de cristalinidad representa a menudo una ventaja al evitar las características transiciones de fases que limitan frecuentemente el rango de ciclabilidad de las fases cristalinas. En esta categoría de materiales electroactivos no cristalinos se encuadran tanto los vidrios como los materiales híbridos que describimos en este artículo. Entre los primeros, los más estudiados han sido los vidrios de vanadato fosfato[2]. Estos vidrios mostraban una reversibilidad superior a sus análogos cristalinos debido precisamente al carácter no cristalino de su estructura. Por otra parte entre los electrodos híbridos destacan los materiales formados por inserción de polianilina entre las capas de xerogeles de $\mathrm{V}_{2} \mathrm{O}_{5}$ [3] .

En este trabajo presentamos la síntesis, caracterización básica y el comportamiento electroquímico de materiales híbridos basados en óxidos $\left(\mathrm{MnO}_{2}, \mathrm{~V}_{2} \mathrm{O}_{5}\right)$ y polímeros conductores (Polianilina PAni, Polipirrol PPi), y materiales vítreos del tipo $\mathrm{V}_{2} \mathrm{O}_{5}-\mathrm{NiO}-\mathrm{TeO}_{2}$; así como su estudio electroquímico como electrodos de inserción y su posible combinación en celdas de ion litio.

\section{PROCEDIMIENTO EXPERIMENTAL}

En el ICMAB hemos preparado diversos tipos de materiales híbridos, de los cuales aquí presentaremos los basados en polipirrol con dióxido de manganeso $\left(\mathrm{PPi} / \mathrm{MnO}_{2}\right)$, y los que combinan polianilina con pentóxido de vanadio (PAni $/ \mathrm{V}_{2} \mathrm{O}_{5}$ ). Por otro lado en el ICV, se desarrollaron los materiales vítreos electroactivos $\left(\mathrm{V}_{2} \mathrm{O}_{5}-\mathrm{NiO}-\mathrm{TeO}_{2}\right)[4]$

\subsection{Reactivos}

La anilina y el pirrol utilizados para la síntesis de híbridos, se adquirieron de la casa Aldrich y se purificaron por medio de una destilación a presión reducida, manteniéndolos en atmósfera de $\mathrm{N}_{2}$ a $4^{\circ} \mathrm{C}$. Los ácidos $\mathrm{H}_{2} \mathrm{SO}_{4} 96 \%, \mathrm{H}_{3} \mathrm{PO}_{4} 67 \%$, fueron suministrados por Panreac. Los reactivos utilizados para la síntesis del gel de pentóxido de vanadio,[5] como la resina 
Dowex 50wx2-100, el $\mathrm{NaVO}_{3} 90 \%$, y el $\left(\mathrm{NH}_{4}\right)_{2} \mathrm{Fe}\left(\mathrm{SO}_{4}\right)_{2} \cdot 6 \mathrm{H}_{2} \mathrm{O}$ 99.9\% se adquirieron de la casa Aldrich y se utilizaron sin purificación previa. $\mathrm{El} \mathrm{KMnO}_{4}$ utilizado para la síntesis del híbrido con manganeso, se obtuvo de Panreac.

Fabricamos cátodos en film del híbrido PAni $/ \mathrm{V}_{2} \mathrm{O}_{5}(52 \% \mathrm{w})$, utilizando Kinar flex (26\%) suministrado por Elfatochem y carbono super P (22\%) adquirido de MMM Belgium, los cuales se secaron a vacío a $40^{\circ} \mathrm{C}$ por 24 horas; el DBP (dibutil ftalato) de la casa Aldrich; y la acetona de Panreac, la cual se secó con un tamiz molecular. El Li metálico utilizado como ánodo se adquirió de Aldrich en forma de cinta con una pureza del 99.9\%, y el electrolito para la batería Selectipur se adquirió de Merck.

\subsection{Caracterización}

Un sistema Carlo Erba CHN EA 1108 se utilizó para los análisis elementales. El contenido de $\mathrm{V}(\mathrm{V})$ en el hidrogel se analizó mediante una valoración potenciométrica descrita anteriormente,[5, 6] utilizando un electrodo de combinación Metrohm of Pt y Ag/ $\mathrm{AgCl}$ y un potenciómetro CRISON. Se utilizó la técnica de absorción atómica para determinar la cantidad de manganeso en los híbridos, en un aparato Unicam PU 9200X. Los análisis FTIR se llevaron a cabo en pastillas con $\mathrm{KBr}$, en un espectrofotómetro FTIR Nicolet 710. Los análisis de difracción de rayos-x en polvo se llevaron a cabo en un difractómetro Rigaku Ru-200B de $2 q=4$ a 60 con una radiación CuKa. Los análisis de SEM se realizaron en un microscopio Hitachi S-570. Para los análisis del área superficial se utilizó un aparato Micrometrics ASAP 200. Para determinar la parte orgánica e inorgánica de los híbridos se utilizó una termobalanza Perkin Elmer ATG-7. Los estudios electroquímicos (análisis en baterías) se llevaron a cabo en celdas swagelock en un potenciostato ARBIN BT2042.

\section{RESULTADOS Y DISCUSIÓN}

\subsection{Híbrido $\mathrm{PPi} / \mathrm{MnO}_{2}$}

Síntesis En esta sección describiremos la síntesis, caracterización y la aplicación en celdas reversibles de Li, de los distintos híbridos obtenidos de $\mathrm{PPi} / \mathrm{MnO}_{2}$.

La formación del híbrido $\mathrm{PPi} / \mathrm{MnO}_{2}$, se llevó a cabo por medio de una polimerización oxidativa del pirrol en medio ácido. El agente oxidante utilizado fue el $\mathrm{KMnO}_{4}$, el cual a su vez se reduce para formar la parte inorgánica del híbrido, y el anión dopante que compensa la carga del polipirrol fue el ion perclorato que proviene del ácido utilizado $\left(\mathrm{HClO}_{4}\right)$. Se utilizó una cantidad de pirrol constante de 0.005 moles, variando la cantidad de $\mathrm{HClO}_{4^{\prime}} \mathrm{KMnO}_{4^{\prime}}$ y el tiempo de reacción; obteniendo así un diseño de experimentos modificado $\mathrm{L}_{9}\left(3^{3}\right)$ de las tablas Taguchi, con un total de 9 experimentos para evaluar la influencia de las variables.[7] Los factores que permanecieron invariables fueron: la temperatura de síntesis de $0^{\circ} \mathrm{C}$, la agitación magnética, y la adición de los reactivos (oxidante a mezcla de pirrol y ácido). En la tabla I se resumen los nueve experimentos diseñados.

El sólido obtenido en cada experimento se filtró, se lavó y se seco a vacío durante 3 días. La única relación o dependencia entre los factores de síntesis que pudimos observar, fue la correlación entre las cantidades de ácido y de permanganato. Para que el híbrido $\mathrm{PPi} / \mathrm{MnO}_{2}$ se forme, se debe tener al menos una relación de $\mathrm{KMnO}_{4}: \mathrm{HClO}_{4}$ de 4:10 moles. Las muestras que cumplieron esta condición y que fueron en definitiva las que
TABla I.- DiseÑo DE EXPERIMENTOS DE TAGUCHI MOdificAdO $\mathrm{L}_{9}\left(3^{3}\right)$, PARA LA EVALUACIÓN DE LOS FACTORES ÓPTIMOS EN LA SÍNTESIS DEL HÍBRIDO PPi $/ \mathrm{MnO}_{2}$.

\begin{tabular}{|l|lr|lr|c|}
\hline Exper. & \multicolumn{2}{|l|}{$\mathbf{H C l O}_{\mathbf{4}} / \mathbf{2 5} \mathbf{~ m l}$} & \multicolumn{2}{|l|}{$\mathbf{K M n O}_{\mathbf{4}} / \mathbf{2 0} \mathbf{~ m l}$} & Tiempo rxn(min) \\
\hline MnPi1 & 0.005 & moles & 0.0025 & moles & 5 \\
MnPi2 & 0.005 & moles & 0.0015 & moles & 10 \\
MnPi3 & 0.005 & moles & 0.001 & moles & 30 \\
MnPi4 & 0.00375 moles & 0.0025 & moles & 10 \\
MnPi5 & 0.00375 moles & 0.0015 & moles & 30 \\
MnPi6 & 0.00375 moles & 0.001 & moles & 5 \\
MnPi7 & 0.0025 & moles & 0.0025 & moles & 30 \\
MnPi8 & 0.0025 & moles & 0.0015 & moles & 5 \\
MnPi9 & 0.0025 & moles & 0.001 & moles & 10 \\
\hline
\end{tabular}

acabamos estudiando y caracterizando fueron las siguientes: MnPi1, MnPi4, MnPi5, MnPi7, MnPi8, y MnPi9.

Los métodos de caracterización utilizados fueron FTIR, análisis termogravimétricos (ATG), análisis elementales y absorción atómica; se llevaron a cabo estudios de difracción de rayos- $X$ en polvo pero no se presentan en este trabajo, ya que estos materiales son amorfos.

FTIR.- Hemos utilizado esta técnica para comprobar la formación del polipirrol en las 9 muestras sintetizadas indicadas en la tabla I. En la figura 1 se representan los espectros infrarrojos de los híbridos que se formaron, donde se indican las bandas correspondientes al polipirrol y al $\mathrm{MnO}_{2}$. De la intensidad de la banda correspondiente al $\mathrm{MnO}_{2}$ a $500 \mathrm{~cm}^{-1}$, podemos predecir aproximadamente el contenido de Mn presente en las respectivas muestras. El orden de mayor a menor contenido de $\mathrm{Mn}$ en las muestras es: MnPi7, MnPi4, MnPi8, MnPi1, MnPi9, y la de menor contenido es la muestra MnPi5.

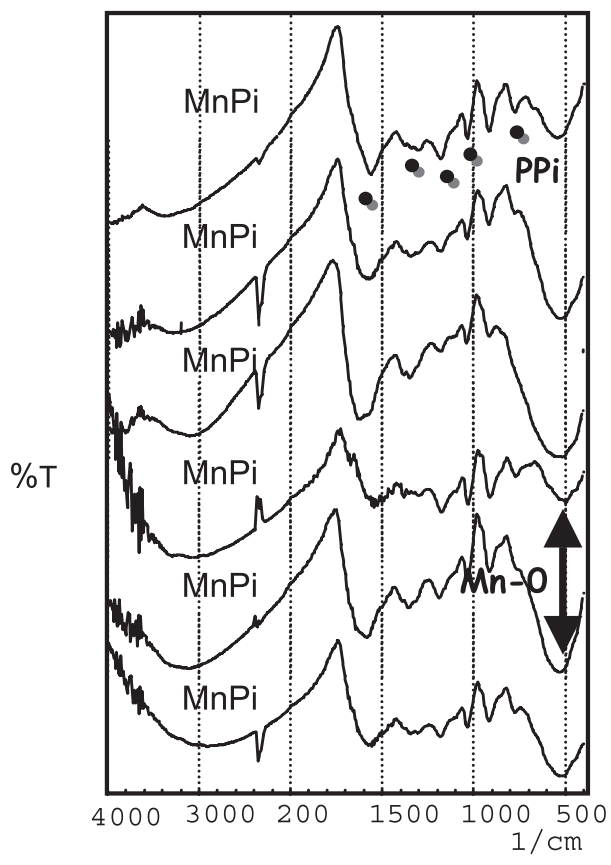

Figura 1.- Espectros infrarrojos de los híbridos de $\mathrm{PPi} / \mathrm{MnO}_{2}$, donde los puntos representan las bandas correspondientes al pirrol y la flecha corresponde a la banda de los estiramientos $\mathrm{Mn}-\mathrm{O}$. 


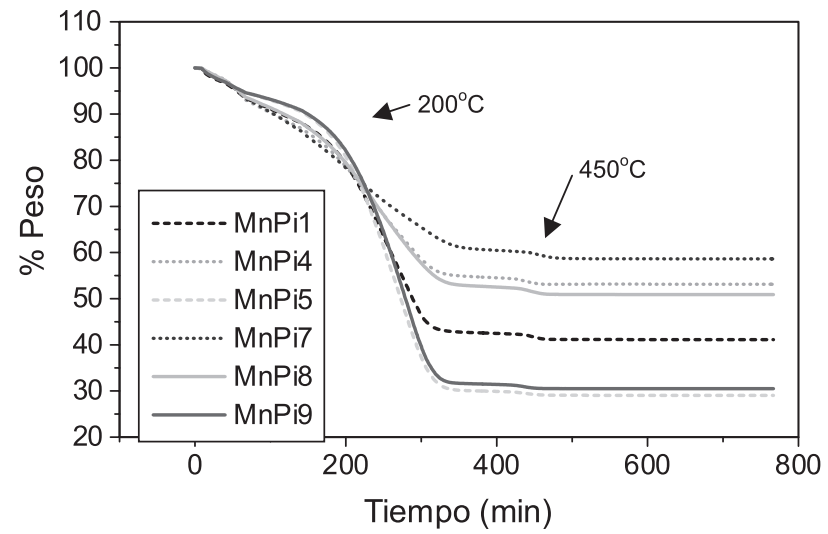

Figura 2.- Análisis termogravimétrico de las muestras del híbrido $\mathrm{PPi} / \mathrm{MnO}_{2}$.

ATG.- Hemos realizado análisis termogravimétricos para determinar los contenidos de materia orgánica y el agua de hidratación ( cuyas pérdidas están parcialmente solapadas) y sobretodo para determinar la materia inorgánica residual. El análisis se ha llevado a cabo en una atmósfera de aire, calentando a una velocidad de $1{ }^{\circ} \mathrm{C} / \mathrm{min}$ hasta una temperatura de $500^{\circ} \mathrm{C}$, y manteniéndola durante 5 horas. En la figura 2 podemos observar que las muestras del híbrido tienen distintos contenidos de materia orgánica y de materia inorgánica. El orden de mayor a menor contenido de materia orgánica es: MnPi5, MnPi9, MnPi8, MnPi4, y el de menor contenido es la muestra MnPi7. Estos datos se correlacionan bastante bien con la estimación del contenido de $\mathrm{MnO}_{2}$ a partir de los espectros infrarrojos (fig. 1). Finalmente, en relación a este análisis, cabe mencionar la pequeña pérdida de peso que tiene lugar en torno a $430^{\circ} \mathrm{C}$ y que se ha asignado previamente a una transformación de fase del $\mathrm{MnO}_{2}$ a $\mathrm{Mn}_{2} \mathrm{O}_{3}$. Este proceso se lleva a cabo acompañado de una liberación de oxígeno.[8, 9]

Absorción atómica.- Los análisis de Mn por absorción atómica se llevaron a cabo sobre el residuo inorgánico, una vez eliminada la parte orgánica y aguas de hidratación de las muestras por ATG. Este análisis es complementario y nos sirvió para determinar la estequiometría de las muestras.

Análisis Elemental.- Se llevó a cabo una serie de análisis de

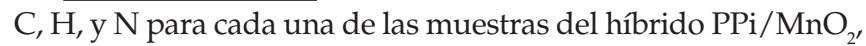
con el objetivo de determinar la composición de la materia orgánica presente. Una vez conocida la cantidad de la parte orgánica perteneciente al PPi en cada muestra, y con los datos obtenidos de ATG, se determinó por diferencia la cantidad de agua de hidratación presente en los híbridos. Además, a partir de los datos obtenidos de absorción atómica, se calculó el contenido de Mn de cada muestra. En la tabla II se resumen los datos calculados y experimentales, para la determinación de la estequiometría de cada híbrido, y están en buen acuerdo con los de los análisis infrarrojos y termogravimétricos, tanto en lo que se refiere al contenido de materia orgánica como a la determinación espectroscópica del contenido de $\mathrm{MnO}_{2}$.

Análisis electroquímico.- En la figura 3 se puede observar la primera descarga de celdas de los híbridos $\mathrm{PPi} / \mathrm{MnO}_{2}$ como cátodos frente a ánodos de litio (el régimen utilizado de carga y descarga fue de C/35). De estos datos podemos concluir que las muestras que resultaron mejores en cuanto a capacidad específica fueron las que tienen mayor contenido de $\mathrm{MnO}_{2}$, (muestras: MnPi4, MnPi7, y MnPi8 con capacidades de $92 \mathrm{Ah} / \mathrm{Kg}, 83 \mathrm{Ah} / \mathrm{Kg}, \mathrm{y} 84 \mathrm{Ah} / \mathrm{Kg}$ respectivamente).

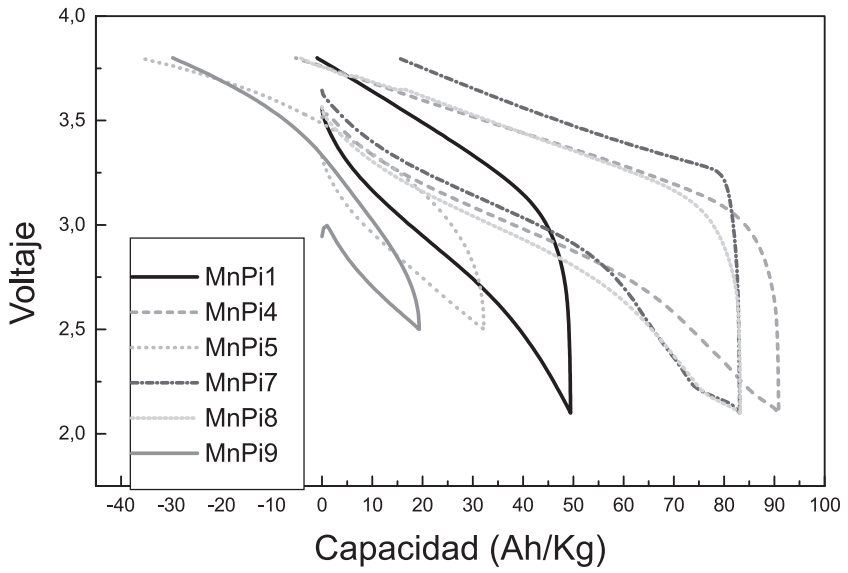

Figura 3.- Gráfico de la primera descarga de los híbridos $\mathrm{PPi} / \mathrm{MnO}_{2}$, a velocidades de carga-descarga lenta $(\mathrm{C} / 35)$.

TABLA II.- TABLA RESUMEN DE LOS ANÁLISIS QUíMICOS Y LAS FÓRMULAS CALCULADAS PARA LOS HÍBRIDOS PPi/ $\mathrm{MnO}_{2}$

\begin{tabular}{|l|c|c|c|c|c|c|l|l|}
\hline & Pi:MnO $_{4}$ & $\% \mathbf{C}$ & $\% \mathbf{N}$ & $\% \mathrm{H}$ & $\% \mathbf{M n}$ & $\mathbf{N} / \mathbf{M n}$ & Fórmula & Muestra \\
\hline Exp. & $2 / 1$ & 36.12 & 10.22 & 2.65 & 28.25 & 1.42 & {$\left[\mathrm{C}_{4} \mathrm{H}_{3} \mathrm{~N}_{1.42} \mathrm{MnO}_{1.84} 0.99 \mathrm{H}_{2} \mathrm{O}\right.$} & MnPi1 \\
Calc. & & 35.05 & 10.22 & 3.23 & 28.23 & & & \\
\hline Exp. & $2 / 1$ & 23.43 & 6.68 & 2.09 & 35.54 & 0.74 & {$\left[\mathrm{C}_{4} \mathrm{H}_{3} \mathrm{~N}_{0.74} \mathrm{MnO}_{1.87} 1.2 \mathrm{H}_{2} \mathrm{O}\right.$} & MnPi4 \\
Calc & & 22.99 & 6.7 & 3.01 & 35.52 & & & \\
\hline Exp. & $10 / 3$ & 43.25 & 12.20 & 2.93 & 19.5 & 2.46 & {$\left[\mathrm{C}_{4} \mathrm{H}_{3} \mathrm{~N}_{2.46} \mathrm{MnO}_{1.85} 2.08 \mathrm{H}_{2} \mathrm{O}\right.$} & MnPi5 \\
Calc & & 41.89 & 12.21 & 4.12 & 17.47 & & & \\
\hline Exp. & $2 / 1$ & 19.6 & 5.58 & 1.93 & 39.99 & 0.55 & {$\left[\mathrm{C}_{4} \mathrm{H}_{3} \mathrm{~N}_{0.55} \mathrm{MnO}_{1.77} 1.02 \mathrm{H}_{2} \mathrm{O}\right.$} & MnPi7 \\
Calc & & 19.22 & 5.6 & 2.7 & 39.97 & & & \\
\hline Exp. & $10 / 3$ & 26.24 & 7.39 & 2.2 & 36.2 & 0.85 & {$\left[\mathrm{C}_{4} \mathrm{H}_{3} \mathrm{~N}_{0.85} \mathrm{MnO}_{1.91} 0.61 \mathrm{H}_{2} \mathrm{O}\right.$} & MnPi8 \\
Calc & & 26.9 & 7.84 & 2.5 & 36.19 & & & \\
\hline Exp. & $5 / 1$ & 40.7 & 12.03 & 2.92 & 21.27 & 2.22 & {$\left[\mathrm{C}_{4} \mathrm{H}_{3} \mathrm{~N}_{2.22} \mathrm{MnO}_{1.79} 1.69 \mathrm{H}_{2} \mathrm{O}\right.$} & MnPi9 \\
Calc & & 41.26 & 12.03 & 3.91 & 21.25 & & & \\
\hline
\end{tabular}

\section{2.- Híbrido PAni/ $\mathrm{V}_{2} \mathrm{O}_{5}$}

Síntesis Hemos llevado a cabo experimentos para la optimización de las propiedades electroquímicas de estos materiales con especial énfasis en los aspectos de microestructura y ciclabilidad que habían sido menos estudiados. Los trabajos anteriores de nuestro grupo sobre este tipo de materiales híbridos[10-13] sugieren una importante influencia de las condiciones de síntesis sobre factores como la microestructura y las propiedades electroquímicas. Aspectos poco estudiados o difíciles de controlar como los procedimientos de mezcla y agitación durante la síntesis o los tratamientos posteriores a la misma pueden influir decisivamente en la actividad y en las prestaciones finales de los materiales híbridos empleados como cátodos en celdas reversibles de Li. Son precisamente estos aspectos los que se han estudiado y optimizado en esta sección.

Para la síntesis del híbrido PAni/ $\mathrm{V}_{2} \mathrm{O}_{5}$ seguimos el procedimiento de polimerización oxidativa de la anilina al reaccionar "in-situ" con el gel de $\mathrm{V}_{2} \mathrm{O}_{5}$ que se describe en la bibliografía.[10] De acuerdo con este trabajo, las condiciones para la sín- 
tesis de un híbrido óptimo comprendían la reacción directa de anilina añadida al gel de $\mathrm{V}_{2} \mathrm{O}_{5}$ (previamente envejecido durante 2 meses) en una relación $3: 1$, a una temperatura de $0^{\circ} \mathrm{C}$ con agitación magnética a 300rpm, con tiempo de síntesis de 120 horas y un tratamiento térmico posterior en oxígeno a $80^{\circ} \mathrm{C}$. Llevamos a cabo por tanto una serie de síntesis con las condiciones antes mencionadas, donde variamos sólo el tipo de agitación y en algunos casos el tiempo, ya que posteriormente se verá que son parámetros correlacionados. En la figura 4 se esquematizan los cuatro distintos tipos de agitación empleados en orden de menor a mayor intensidad o turbulencia. Los ensayos con agitación más intensa (A3 y A4) permitieron el desarrollo de síntesis en un tiempo menor, por lo que decidimos variar el tiempo de síntesis y estudiar su efecto sobre los materiales obtenidos. Además en el tipo de agitación A4 hicimos otra modificación impuesta por el método (aumento al doble de los volúmenes usados y sólo a temperatura ambiente). En la denominación de las muestras, el primer número acompañado por la letra A indica el método de agitación utilizado en la síntesis, y el segundo número indica el número de días que dura la síntesis (A33= A3 agitación mecanica tipo turbohélice, 3 días). Las siglas TO significan un tratamiento adicional a la muestra, en oxígeno a $80^{\circ} \mathrm{C}$ durante 5 horas.

Análisis electroquímico.- En la figura 5 presentamos un análisis comparativo de la capacidad de descarga de los diferentes híbridos (obtenidos por distintos métodos de agitación) en los primeros 15 ciclos a un régimen de $\mathrm{C} / 6$. La gráfica marcada como "referencia" corresponde a los mejores resultados anteriores obtenidos por nuestro grupo. Como se puede observar dentro de los mejores cátodos están aquellos preparados con una agitación más intensa (como las muestras de la serie A4 y algunas de la serie A3 ). Si bien parece existir un tiempo óptimo para cada serie, por encima del cual los resultados de capacidad específica empeoran.

Con los procedimientos de síntesis que describimos aquí, es decir, con agitación vigorosa y mezcla de los reactivos en aire durante el crecimiento del polímero híbrido, hemos observado que el tratamiento oxidante que mejoraba los valores de carga específica en materiales preparados con agitación menos intensa, se hace innecesario. Esto se debe, con toda probabilidad a que la turbulencia inducida por agitación mecánica da lugar a una mayor interacción de la mezcla de reacción con el aire que equivale al tratamiento oxidante posterior. De hecho, con una sola excepción, todos los materiales sintetizados que describimos aquí empeoraron su carga específica al someterlos a tratamientos en $\mathrm{O}_{2}$ a $80^{\circ} \mathrm{C}$. La excepción es la muestra óptima $\mathrm{A} 41 \mathrm{TO}$, que es precisamente la muestra preparada durante un tiempo más corto de la serie A4.

Difracción de rayos x.- Los difractogramas de algunos de nuestros híbridos seleccionados se presentan en la Figura 6. En general todos ellos presentan picos derivados del $\mathrm{V}_{2} \mathrm{O}_{5}$ solapados con amplias bandas de dispersión difusa que se pueden asociar a la PAni. La cristalinidad es menor para la muestra de referencia y A33, que para las muestras A43, A42 y A41TO. El pico más intenso a ángulos bajos corresponde a la reflexión 001 que indica un espaciado de $14.8 \AA$ entre las capas de la estructura de la matriz de $\mathrm{V}_{2} \mathrm{O}_{5}$ expandida con la inserción del polímero.[14] Esta expansión (el espaciado del $\mathrm{V}_{2} \mathrm{O}_{5}$ es originalmente de $11.5 \AA$ ) representa la eliminación de una capa de agua del espacio interlaminar (contracción de 2.8 Å) compensada por la inserción de una monocapa de PAni (expansión de unos 6 Å).[11]

SEM.- Hemos podido observar por microscopia electrónica de barrido la microestructura de estos híbridos, donde las
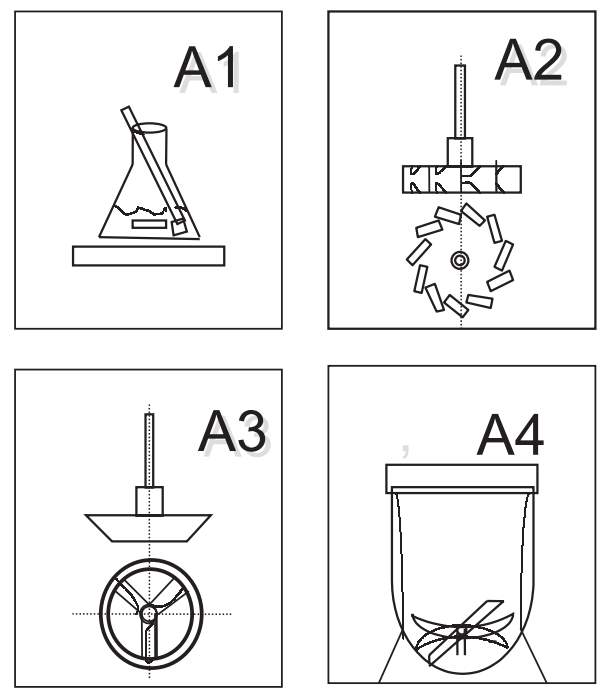

Figura 4.- Distintos tipos de agitación empleados. A1) agitación magnética con burbujeo de aire, A2)agitación mecánica tipo turbina, A3) agitación mecánica tipo turbohélice, y A4)agitación en mezcladora mecánica. Están descritas en orden de menor a mayor movilidad.

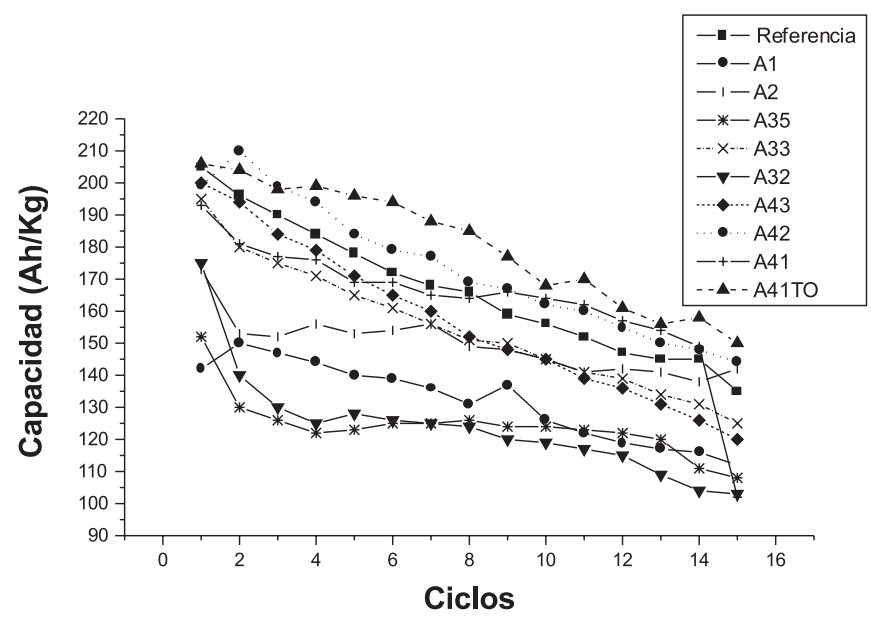

Figura 5.- Representación gráfica de la Capacidad específica, a un régimen de descarga de $\mathrm{C} / 6$, vs. ciclos de descarga para diversas muestras del híbrido PAni $/ \mathrm{V}_{2} \mathrm{O}_{5}$ obtenidos con diferentes tipos de agitación.

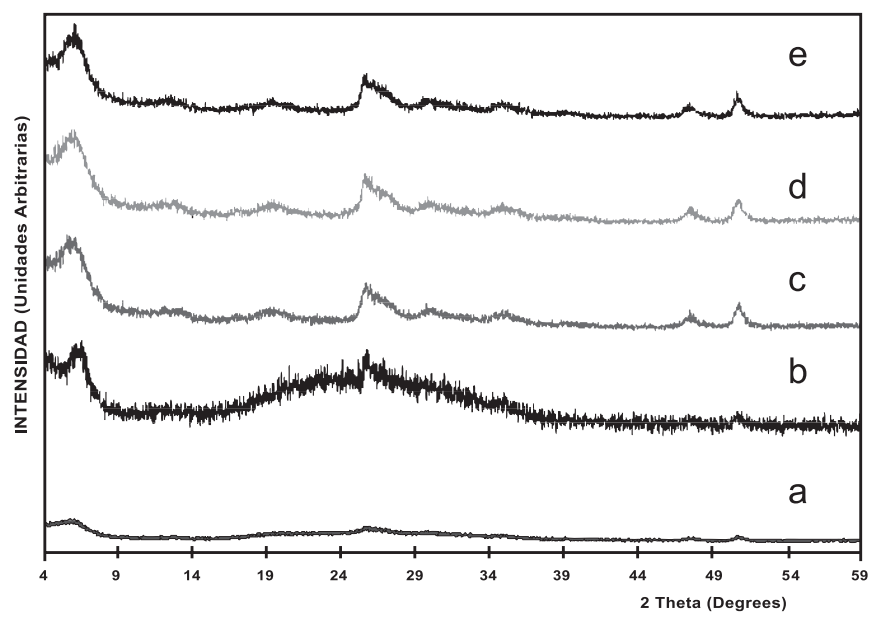

Figura 6.- Difractogramas de los híbridos $\mathrm{PAni} / \mathrm{V}_{2} \mathrm{O}_{5}$ seleccionados a)referencia, b)A33, c)A43, d)A42, y e)A41TO 
muestras de la serie A4 tienen un tamaño de grano menor. Esto podría explicar en parte su mejor comportamiento como cátodo de inserción de litio. Es decir el tamaño de partícula menor implica un área superficial activa mayor y como consecuencia una mejora en sus características electroquímicas.

BET.- Hemos analizado nuestras muestras por BET para determinar el área superficial. En la figura 7 podemos observar una gráfica que nos relaciona el área superficial con el tiempo de reacción, donde encontramos cambios significativos. Se observa una evolución lineal del área superficial, que aumenta a medida que se aumenta el tiempo de agitación de la mezcla de reacción (A4). Encontramos un área superficial mayor para la muestra A43 de $99 \mathrm{~m}^{2} / \mathrm{g}$.

Determinación de la estequiometría.- hemos determinado la composición química de los mejores híbridos de la serie A4, mediante análisis elementales y ATG. Por termogravimetría hemos determinado la cantidad de la parte inorgánica $\left(\mathrm{V}_{2} \mathrm{O}_{5}\right)$, y la parte orgánica solapada por el agua de hidratación. Los análisis elementales nos han ayudado a determinar la composición de la parte orgánica y por diferencia con ATG el agua de hidratación. En la tabla III se resumen los datos experimentales y calculados, que presentan un buen acuerdo y que confirman las composiciones propuestas. Cabe destacar que con los procedimientos de síntesis descritos en este trabajo se obtienen híbridos con mayor contenido de polianilina por unidad de $\mathrm{V}_{2} \mathrm{O}_{5}$ aunque sin una expansión adicional de la red del óxido.

Estudios de ciclabilidad de los híbridos. Finalmente hemos llevado a cabo estudios de los mejores materiales híbridos en celdas reversibles frente a ánodos de litio y hemos evaluado la ciclabilidad de las mejores muestras del híbrido PAni $/ \mathrm{V}_{2} \mathrm{O}_{5}$, serie A4 (A43, A42, y A41TO). El estudio electroquímico se realizó en un rango de voltaje de 3.8-2.1V, bajo un régimen de carga-descarga de $\mathrm{C} / 6$.

Los datos que se muestran se obtuvieron con celdas en las que los cátodos híbridos se utilizaron en forma de film frente a un ánodo metálico de litio. Además del procedimiento de secado habitual (secado a vacío durante 3 días), estos materiales se sometieron a un secado a vacío a $30^{\circ} \mathrm{C}$ durante 12 horas, para eliminar completamente el agua de hidratación. El film híbrido se fabricó utilizando las cantidades correspondientes de sus componentes (carbono super $\mathrm{p}$, kinar flex, y el híbrido) suspendidos en acetona, con un $50 \%$ en peso de DBP del total de la mezcla sólida, se agito hasta obtener una mezcla viscosa que se extendió sobre una superficie plana. Al secarse el film se lavó con éter para eliminar el DBP y los oligómeros no deseados.

En la figura 8 se puede comparar la ciclabilidad de estos híbridos de la serie A4. Se observa que el híbrido A43 tiene una mayor capacidad específica inicial comparado con A42 y A41TO. Por otra parte, a pesar de las distintas pendientes de pérdida de capacidad en los ciclos iniciales, la capacidad específica final después de 100 ciclos tiende a estabilizarse y converge en los tres casos en torno a valores de $60 \mathrm{Ah} / \mathrm{Kg}$. Este valor es excesivamente bajo y sugiere la necesidad de seguir investigando para llegar a comprender el origen de la pérdida de capacidad y optimizar el comportamiento de estos materiales. En parte con este fin se llevaron a cabo ensayos de celdas de ion litio (véase más abajo) para poder intentar asignar la pérdida de capacidad específica. Estos híbridos óptimos tienen mayor capacidad en el primer ciclo que los híbridos que se presentan en la Fig. 5, debido al secado adicional y a que los cátodos utilizados son en forma de film.

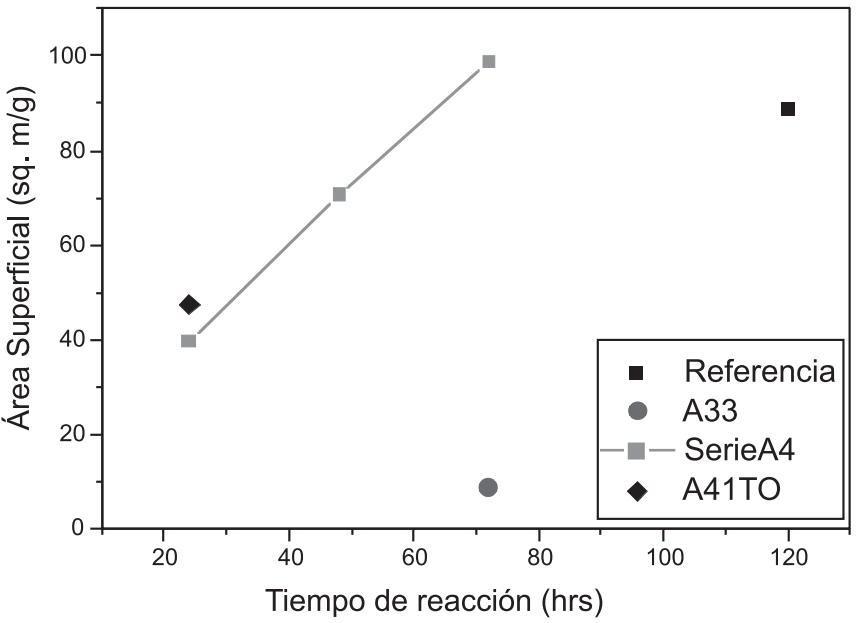

Figura 7.- Gráfica de la serie de experimentos A4, tiempo vs. área superficial (BET). Incluyendo los híbridos de referencia, A33, y A41TO.

TABLA III.- RESUMEN DE LOS DATOS OBTENIDOS DEL ANÁLISIS ELEMENTAL Y ANÁLISIS TERMOGRAVIMÉTRICO PARA DETERMINAR LA ESTEQUIOMETRÍA Y PESO FÓRMULA DE LOS HÍBRIDOS PAni/ $\mathrm{V}_{2} \mathrm{O}_{5}$.

\begin{tabular}{|l|l|l|l|l|l|l|l|l|}
\hline & $\mathrm{An}: \mathrm{V}_{2} \mathrm{O}_{5}$ & $\% \mathrm{C}$ & $\% \mathrm{~N}$ & $\% \mathrm{H}$ & $\% \mathrm{~V}_{2} \mathrm{O}_{5}$ & $\mathrm{~N} / \mathrm{V}_{2} \mathrm{O}_{5}$ & Fórmula & Muestra \\
\hline Exp. & $3: 1$ & 26.81 & 5.1 & 1.73 & 63 & 1.05 & $\left(\mathrm{C}_{6} \mathrm{H}_{5} \mathrm{~N}\right)_{1.05} \mathrm{~V}_{2} \mathrm{O}_{5} 0.6 \mathrm{H}_{2} \mathrm{O}$ & A41T0 \\
Calc. & & 26.24 & 5.1 & 2.25 & 63.08 & & & \\
\hline Exp. & $3: 1$ & 24.75 & 4.53 & 1.61 & 65.62 & 0.9 & $\left(\mathrm{C}_{6} \mathrm{H}_{5} \mathrm{~N}_{0.9} \mathrm{~V}_{2} \mathrm{O}_{5} 0.76 \mathrm{H}_{2} \mathrm{O}\right.$ & A42 \\
Calc. & & 23.36 & 4.54 & 2.18 & 65.52 & & & \\
\hline Exp. & $3: 1$ & 26.20 & 4.98 & 1.85 & 61.9 & 1.06 & $\left(\mathrm{C}_{6} \mathrm{H}_{5} \mathrm{~N}\right)_{1.06} \mathrm{~V}_{2} \mathrm{O}_{5} \mathrm{H}_{2} \mathrm{O}$ & A43 \\
Calc. & & 25.76 & 5.0 & 2.48 & 61.35 & & & \\
\hline
\end{tabular}

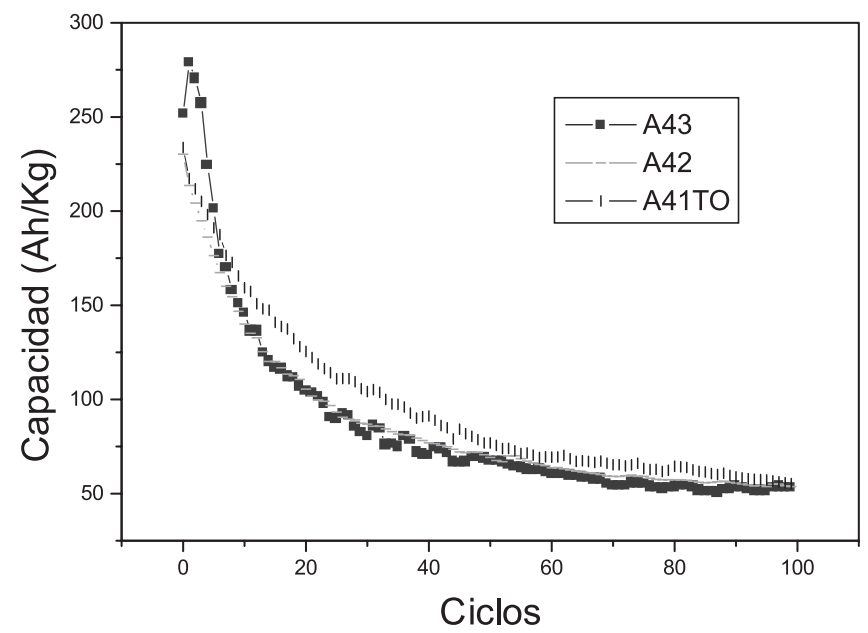

Figura 8.- Comparación de los cátodos de PAni $/ \mathrm{V}_{2} \mathrm{O}_{5}$ de la serie sintetizada de la forma A4, en 100 ciclos sucesivos de carga-descarga. 


\subsection{Vidrios $\mathrm{V}_{2} \mathrm{O}_{5}-\mathrm{NiO}-\mathrm{TeO}_{2}$}

Síntesis Las proporciones de los óxidos constituyentes de los vidrios oscilaron entre $0-20 \mathrm{~mol} \%$ de $\mathrm{NiO}, 15-30 \mathrm{~mol} \%$ de $\mathrm{TeO}_{2}$ y 55 a $70 \mathrm{~mol} \%$ de $\mathrm{V}_{2} \mathrm{O}_{5}$. Estos dos últimos óxidos actuaron como formadores de red vítrea. Los vidrios fueron sintetizados a partir de los óxidos de partida (MERCK, pureza analítica) de alto grado de pureza. Los polvos se mezclaron y homogeneizaron en molino de bolas durante dos horas y después se fundieron en crisol de platino a $950^{\circ} \mathrm{C}$ durante 4 horas. El vidrio fundido se coló en moldes apropiados hasta temperatura ambiente en aire. El vidrio consolidado presentaba color negro y se observó una gran homogeneidad, ya que no exhibía ningún defecto como burbujas o núcleos de cristalización, lo cual se confirmó por difracción de rayos $X$ (Difractómetro Siemens D-5000, radiación $\mathrm{CuK}_{\alpha} 1$ ). La composición vítrea fue sometida a una molienda de cuatro horas, y

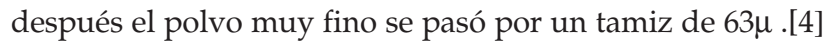

Caracterización electroquímica. El material vítreo específicamente estudiado es de composición $60 \mathrm{~V}_{2} \mathrm{O}_{5}: 15 \mathrm{NiO}_{2} 25 \mathrm{TeO}_{2^{\prime}}$ que es el óptimo en cuanto a mejor capacidad de descarga en el 1er. ciclo [4]. La Figura 9a Muestra los ensayos de caracterización electroquímica de este material vítreo en celdas electroquímicas reversibles frente a ánodos de litio. En primer lugar destaca la diferencia de carga específica entre la primera descarga (420 Ah/Kg hasta $1.5 \mathrm{~V})$ y los ciclos subsiguientes. Se trata de una capacidad irreversible indeseable pero presente en la mayor parte de materiales anódicos y que en este caso es de unos $160 \mathrm{Ah} / \mathrm{Kg}$. La primera recarga, hasta $4.5 \mathrm{~V}$ es incapaz de regenerar toda la carga, si bien consigue inyectar 260 $\mathrm{Ah} / \mathrm{Kg}$ que sí se recuperan en los siguientes ciclos de forma prácticamente reversible. Concretamente, tal y como se representa en la figura 9b, al cabo de 25 ciclos de carga y descarga, la carga específica aumenta en los primeros ciclos hasta 290Ah/Kg para después disminuir lentamente hasta 210 $\mathrm{Ah} / \mathrm{Kg}$.

Celdas reversible de ion litio: Las celdas de ion litio se montaron y estudiaron con el doble propósito de establecer su viabilidad pero también de estudiar la descarga de los electrodos híbridos de $\mathrm{V}_{2} \mathrm{O}_{5}$ frente a ánodos diferentes del litio metálico, con el fin de establecer los factores que contribuyen a la pérdida de carga en estos electrodos híbridos. Para el montaje de estas celdas se procedió previamente a un tratamiento reductor de los vidrios $\mathrm{V}_{2} \mathrm{O}_{5}-\mathrm{NiO}-\mathrm{TeO}_{2}$ frente a litio (equivalente a su descarga como electrodo positivo frente a litio) para después incorporar los vidrios reducidos como ánodos en celdas reversibles frente a cátodos de PAni $/ \mathrm{V}_{2} \mathrm{O}_{5}$. La figura 10a muestra los primeros ciclos de descarga de estas celdas de ion

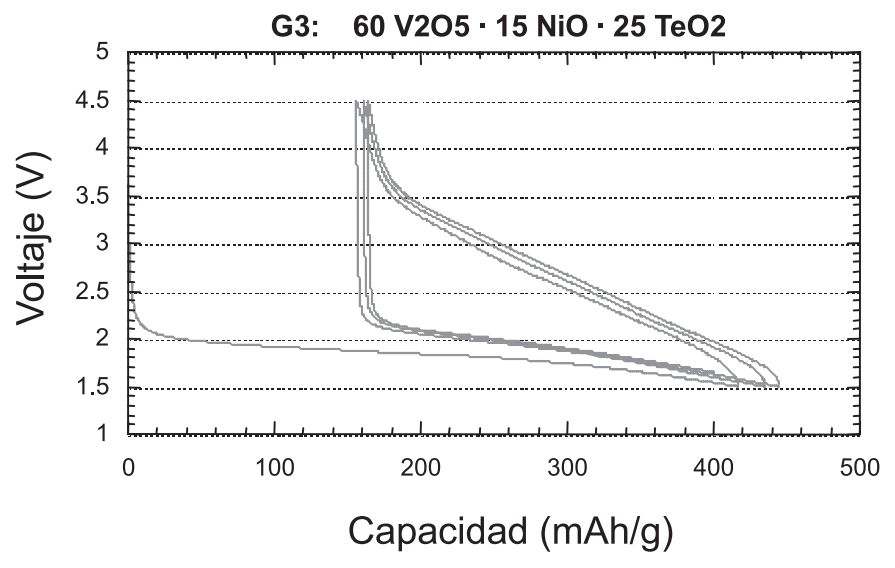

Figura 9a.- Curvas de carga y descarga de electrodos del vidrio $60 \mathrm{~V}_{2} \mathrm{O}_{5}: 15 \mathrm{NiO}: 25 \mathrm{TeO}_{2}$ frente a ánodos de litio.

Hvid3

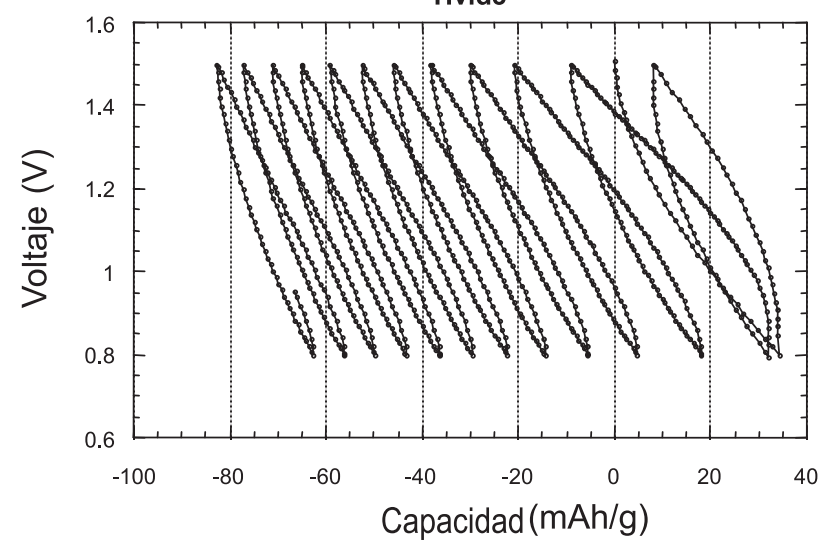

Figura 10a.- Ciclos de carga y descarga para la celda de ion litio formada por un cátodo híbrido $\mathrm{PAni} / \mathrm{V}_{2} \mathrm{O}_{5} \mathrm{y}$ un ánodo vítreo $60 \mathrm{~V}_{2} \mathrm{O}_{5}: 15 \mathrm{NiO}: 25 \mathrm{TeO}_{2}$.

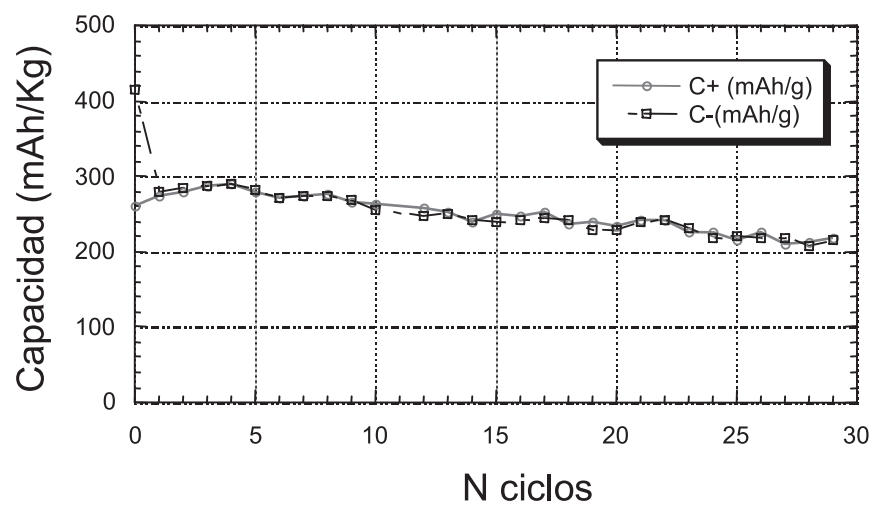

Figura 9b.- Evolución de la carga específica de la celda $60 \mathrm{~V}_{2} \mathrm{O}_{5}: 15 \mathrm{NiO}: 25 \mathrm{TeO}_{2}$ frente a litio en función del número de ciclos.

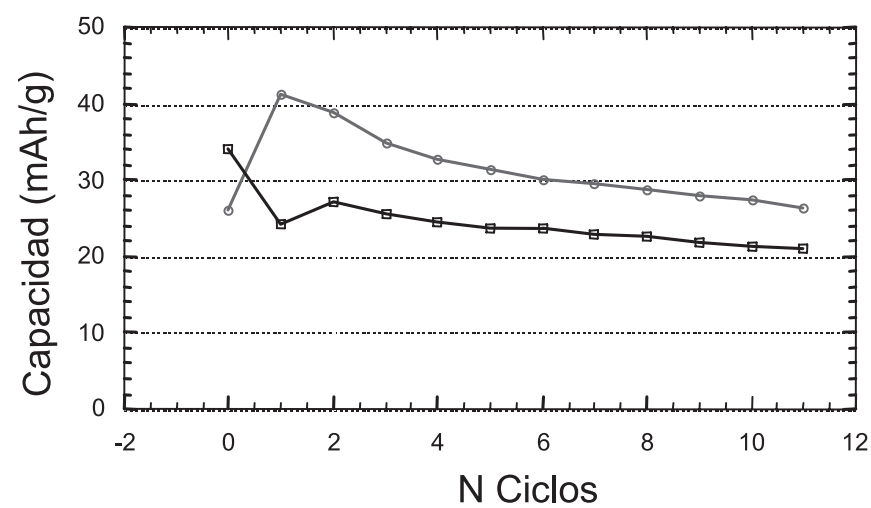

Figura 10b.- Evolución de la carga específica con los ciclos para la celda de ión litio. 
litio en los que se observa una reducida carga específica, así como una diferencia mantenida a lo largo de los ciclos entre la carga inyectada durante la recarga (sistemáticamente superior) y la carga recuperada en cada descarga, lo cual sugiere la presencia de procesos de oxidación irreversibles en el cátodo durante la recarga de la celda. En la figura $10 \mathrm{~b}$ podemos observar la evolución de la carga específica o capacidad en los ciclos sucesivos de carga-descarga. Por otra parte, el potencial relativamente elevado de los electrodos vítreos y su elevada polarización (Figura 9a) dan lugar a una celda de ion litio con un voltaje relativamente reducido, (ciclada entre 1.5 y $0.8 \mathrm{~V}$ ).

\section{CONCLUSIONES}

Hemos desarrollado un procedimiento "in-situ" para la síntesis simultanea del polímero conductor y el óxido en el material híbrido $\mathrm{PPi} / \mathrm{MnO}_{2}$, en la que la polimerización oxidativa del pirrol tiene lugar a la vez que la reducción del anión $\mathrm{MnO}_{4}^{-}$a $\mathrm{MnO}_{2}$. Hemos comprobado la formación de $\mathrm{PPi} / \mathrm{MnO}_{2}$ mediante espectroscopia infrarroja, y hemos determinado la composición de los materiales híbrido obtenidos mediante ATG y análisis químicos obteniendo materiales de fórmulas $\left(\mathrm{C}_{4} \mathrm{H}_{3} \mathrm{~N}\right)_{\mathrm{x}} \mathrm{MnO}_{2-\mathrm{y}} \mathrm{zH} 2 \mathrm{O}$ donde $0.5<\mathrm{x}<2.5,0.1<\mathrm{y}<0.2$, $0.6<\mathrm{z}<2$. Al actuar estos híbridos de $\mathrm{PPi} / \mathrm{MnO}_{2}$ como cátodos de inserción de $\mathrm{Li}$, vemos que cuanto mayor es el contenido de Mn mayor es la carga específica inicial llegando a valores moderados de $90 \mathrm{Ah} / \mathrm{Kg}$.

En cuanto al hibrido de PAni $/ \mathrm{V}_{2} \mathrm{O}_{5}$ hemos determinado factores adicionales de importancia que influyen en la síntesis y que afectan asimismo a sus propiedades electroquímicas. En este sentido cabe destacar los estudios del efecto del tipo de agitación en las mezclas de reacción, que han influido sobre la microestructura y área superficial de los materiales, eliminando los post-tratamientos oxidativos, lo cual afecta directamente a su electroactividad. Los valores de carga específica inicial obtenidos para ciclos de carga-descarga a velocidades de C/6 llegan a alcanzar $280 \mathrm{Ah} / \mathrm{Kg}$, pero su ciclabilidad es limitada (al cabo de 100 ciclos se ve disminuida hasta alcanzar una capacidad estable de $60 \mathrm{Ah} / \mathrm{kg}$ ) y necesita ser mejorada. Por otra parte, la asociación de cátodos híbridos con ánodos vítreos en el sistema $\mathrm{V}_{2} \mathrm{O}_{5}-\mathrm{NiO}-\mathrm{TeO}_{2}$ permite asociar los problemas de ciclabilidad a factores intrínsecos de los cátodos híbridos empleados.
Por otra parte, desde un punto de vista básico, este trabajo ha aportado nuevos datos sobre la inserción de PAni en los geles de $\mathrm{V}_{2} \mathrm{O}_{5}$. Estos nuevos derivados híbridos PAni/ $\mathrm{V}_{2} \mathrm{O}_{5}$ , presentan un mayor contenido de PAni por unidad de $\mathrm{V}_{2} \mathrm{O}_{5}$ que los publicados hasta ahora, pero además este contenido resulta compatible con una expansión moderada del espaciado interlaminar del gel, correspondiente a la inserción de una monocapa de polímero.

\section{BIBLIOGRAFÍA}

1. P. Gomez-Romero,"Hybrid Organic-Inorganic Materials- In search of Synergic Activity.", Adv. Mater. 13, 163-174 (2001).

2. P. Prasad, S. Sainath, S. Radhakrishna, "Experimental Techniques for Characterization of Fast Ion Conducting Materials", Anal. Tech. Mater. Charact. Proc. Int. Workshop, 279-317 (1987).

3. M. Lira-Cantú, A.K. Cuentas-Gallegos, G. Torres-Gómez, P. Gómez-Romero, "Hybrid Electrodes Based on Polyaniline $/ \mathrm{V}_{2} \mathrm{O}_{5}$ for the Development of Plastic Lithium Batteries", Bol. Soc. Esp. Ceram. Vidiro 39, 386-390 (2000)

4. J. M. Amarilla, M. T. Colomer, J. L. Acosta, E. Morales, J. R. Jurado,"Electrochemical Properties of Electrodes Based on Innovative Vanadium-Tellurite Glasses in a Lithium Cell.", Proc. Electrochem. Soc. 97, 233-240 (1997).

5. J. Livage,"Vanadium Pentoxide Gels", Chem. Mater. 3, 578-593 (1991).

6. I. M. Kolthoff, E. B. Sandell, E. J. Meehan, S. Bruckenstein, "Análisis Químico Cuantitativo.", Buenos Aires, 1969.

7. G. Taguchi, S. Konishi, "Arreglos Ortogonales y Gráficas Lineales: Herramientas para la Ingeniería de Calidad.", ASI International, Monterrey N.L., 1991.

8. H. Yoneyama, A. Kishimoto, S. Kawabata,"Charge-Discharge Properties of Polypyrrole Films Containing Manganese Dioxide Particles", J. Chem. Soc., Chem. Commun, 986-987 (1991).

9. H. Gemeay, H. Nishiyama, S. Kuwabata, H. Yoneyama,"Chemical Preparation of Manganese Dioxide/Polypyrrol Composites and Their Use as Cathode Active Materials for Rechargeable Lithium Batteries.", J. Electrochem. Soc. 142, 4190-4195 (1995).

10. M. Lira-Cantú, Tesis Doctoral, Universitat Autònoma de Barcelona, Barcelona, 1997, p. 244.

11. M. Lira-Cantú, P. Gomez-Romero,"The Polyaniline-V2O5 System: Improvement as Insertion Electrode in Lithium Batteries.", International Journal of Inorganic Materials. 1, 111-116 (1999).

12. M. Lira-Cantú, P. Gómez-Romero,"Synthesis and Characterization of Intercalate Phases in the Organic-Inorganic Polyaniline/V2O5 System.", Solid State Chemistry 147, 601-608 (1999).

13. M. Lira-Cantú, P. Gomez-Romero,"The Organic-Inorganic Polyaniline/V2O5 System. Application as a High-Capacity Hybrid Cathode for Rechargeable Lithium Batteries.", J. Electrochem Soc. 146, 2029-2033 (1999).

14. F. Leroux, B. E. Koene, L. F. Nazar,"Electrochemical Lithium Intercalation into a Polyaniline/V2O5 Nanocomposite", J. Electrochem. Soc. 143, L181L183 (1996).

Recibido: 31.05 .01

Aceptado: 10.12.01 